\section{Infliximab reduces BMD loss in patients with rheumatoid arthritis}

Infliximab retards progression of radiographically visible joint damage in patients with rheumatoid arthritis (RA). Vis and colleagues investigated whether infliximab can also prevent osteoporosis in the spine and hip, and periarticular osteoporosis and erosions in the hands.

This 1-year trial included 138 consecutive patients with RA. Intravenous infusions of $3 \mathrm{mg} / \mathrm{kg}$ body weight infliximab were given at 0, 2, 6 and 14 weeks, and every 8 weeks thereafter (doses were increased to $7.5 \mathrm{mg} / \mathrm{kg}$ body weight in patients who responded inadequately). Bone mineral density (BMD) of the spine, hip and hands were measured at baseline and 1 year. Serum markers of bone metabolism were measured at 0, 14, 28 and 46 weeks.

In the 102 patients who completed the study, mean Disease Activity Scores in 28 joints decreased (from 5.5 at baseline to 3.5 after 40 weeks). Spine and hip BMD were unchanged during infliximab treatment; however, compared with baseline, hand BMD decreased significantly, by $0.8 \%$ at 48 weeks $(P<0.05)$, which indicated that these infliximab doses did not completely halt bone loss in the hands. Bone resorption, activation and differentiation of osteoclasts were markedly decreased compared with baseline at all time points. The decreased bone resorption was associated with decreased disease activity and reduced $\mathrm{C}$-reactive protein levels during the first 14 weeks.

The authors conclude that in infliximabtreated patients with RA, the arrest of BMD loss in the spine and hip is secondary to a decrease in disease activity.

Original article Vis M et al. (2006) Evaluation of bone mineral density, bone metabolism, osteoprotegerin and receptor activator of the NFkB ligand serum levels during treatment with infliximab in patients with rheumatoid arthritis. Ann Rheum Dis 65: 1495-1499

\section{$T_{H} 17$ cells are a potential therapeutic target in autoimmune arthritis}

www.nature.com/clinicalpractice/rheum

In autoimmune arthritis, activated type $1 \mathrm{~T}$ helper $\left(T_{H} 1\right)$ lymphocytes were previously thought to cause bone destruction mediated by osteoclasts. Paradoxically, however, $T_{H} 1$ lymphocytes produce interferon- $\gamma$, which inhibits osteoclastogenesis. Bone destruction might, therefore, be attributable to a different subset of CD4 ${ }^{+}$ $T_{H}$ cells, with a specific cytokine profile. Sato and colleagues explored the effects of various T-cell subsets on osteoclast differentiation, and found that interleukin-(IL)-17-producing $\mathrm{CD}^{+} \mathrm{T}_{\mathrm{H}}$ cells $\left(T_{H} 17\right.$ cells) were the only osteoclastogenic T-cell lineage characterized to date.

Sato and colleagues' experiments in vitro and in mouse models revealed that the IL-23IL-17 axis was crucial to the bone-destruction phase of autoimmune arthritis, as well as to the onset phase. IL-23 stimulated both proliferation of $T_{H} 17$ cells and production of IL-17, which in turn induced expression of the osteoclastogenic cytokine receptor activator of NF- $\mathrm{kB}$ ligand (RANKL) on osteoblasts. IL-17 facilitated the release of proinflammatory cytokines, which caused local inflammation; RANKL induced by these proinflammatory cytokines also (indirectly) promoted differentiation of osteoclasts. Sato and colleagues suggest, therefore, that infiltration of $\mathrm{T}_{\mathrm{H}} 17$ cells into autoimmune arthritic lesions in the synovium tips the microenvironment toward osteoclastogenesis.

The authors report that their results provide a molecular rationale for targeting the IL-23-IL-17 axis in autoimmune arthritis. Therapies that prevent IL-17 release from $\mathrm{T}_{\mathrm{H}} 17$ cells might ameliorate the bone destruction that is associated with T-cell activation in this disease.

Original article Sato K et al. (2006) Th17 functions as an osteoclastogenic helper $\mathrm{T}$ cell subset that links $\mathrm{T}$ cell activation and bone destruction. J Exp Med 203: 2673-2682 